\title{
Index of Refraction and Particle Size as Factors in the Infrared Spectrophotometry of Polyvinyl Chloride
}

\author{
Mary Reiney Harvey, James E. Stewart, and Bernard G. Achhammer
}

\begin{abstract}
The scope of solid-phase infrared spectroscopy is broadened by the elimination of particle size restrictions heretofore placed on the sample. This applies only, however, to samples whose indices of refraction change only slightly with change in wavelength and can be matched to the indices of refraction of the suspending medium.

The potassium bromide pellet technique has been applied to the infrared spectral measurement of relatively large, solid particles (up to 44 microns diameter) of polyvinyl chloride, thus providing a technique for obtaining the infrared spectrum of poly vinyl chloride without subjecting the polymer to degrading forces. A near-match in the indices of refraction of potassium bromide and polyvinyl chloride is apparently responsible for the wellresolved, scatter-free spectrum obtained. Other imbedding salts having different indices of refraction were used to demonstrate the necessity of matching the indices of refraction of medium and sample when the sample particles are relatively large. The measurement of potassium bromide pellets containing polyvinyl chloride of various particle sizes showed that even very large particles (up to 110 microns) will yield characteristic spectra when the indices of refraction of sample and medium are matched. Infrared spectra of nylon and paper fibers imbedded in potassium bromide were obtained; these spectra demonstrate the the feasibility of this method for use with other materials. Equipment developed to make suitable pellets is described.
\end{abstract}

\section{Introduction}

Solid-phase infrared spectral measurements have been the subject of wide interest in recent years. The potassium bromide pellet method developed independently by Sister Miriam Michael Stimson [1] ${ }^{1}$ and $\mathrm{U}$ Schiedt [2] has received, in particular, widespread attention. ${ }^{2}$ This paper describes a further development of this technique to render it applicable to samples of high polymers of relatively large particle sizs.

Most solid phase methods $[3,4,5,6,7]$ require that the material being; tudied be soluble or of a very fine particle size (smaller than the wavelength of incident radiant energy), or the methods employ heat or grinding. Also, a solid piece might be molded, which would require heat and pressure when high polymers are under consideration. Polyvinyl chloride cannot be subjected to heat or grinding without degrading the polymer. In addition, it is not soluble in infrared transmitting solvents and the particle size cannot be reduced without concomitant breakdown of the polymer. As a result, the potassium bromide pellet method has been modified to allow the inclusion of relatively large solid particles of polyvinyl chloride. This adaptation is feasible due to the matching of the indices of refraction of the two materials.

\section{Materials}

The polyvinyl chloride sample used predominantly in this investigation and referred to as polymer 101 is a commercial polymer. ${ }^{3}$ This polymer is a

\footnotetext{
1 Figures in brackets indicate the literature references at the end of this paper. 2 A session of the 1954 Pittsburgh Conference on Analytical Chemistry and Applied Spectroscopy was devoted to discussion of the potassium bromide pellet technique. The work described in this report was partially presented in a brief discussion at that time.

${ }_{3}^{3}$ Geon 101, kindly supplied by the B. F. Goodrich Chemical ${ }^{\circ} \mathrm{Co}$.
}

hard, horny, tough, and abrasion-resistant thermoplastic material. It has outstanding resistance to chemicals and oils.

It is a relatively insoluble substance, being soluble in only a few solvents. Solvents include cyclohexanone, tetrahydrofuran, dimethyl formamide, and mixtures of tetrahydrofuran, methyl ethyl ketone, and toluene. Thin films were cast from a solution of the polymer in cyclohexanone, but their infrared spectra indicated that appreciable solvent was retained. In addition, both cyclohexanone and tetrahydrofuran tend to form peroxides in the presence of air [8], making their use as a solvent even more undesirable. Dimethyl formamide has a boiling point in the range of that of cyclohexanone and, therefore, would probably also be retained by a film cast from a solution composed primarily of dimethyl formamide.

The particle size of the bulk powder was examined. A small portion passed the U. S. Standard No. 325 sieve, which has a nominal opening of $44 \mu$. Increasingly larger amounts were collected by wetsieving through coarser sieves of the series; the greatest fraction consisted of particles passing sieve No. 120 and retained on No. 170, that is, having a nominal diameter between 88 and $125 \mu$. There was very little powder retained on sieve No. 120. The Roller analyzer [9] was employed in an attempt to fractionate the powder that passed the No. 325 sieve. When the analyzer was set to collect particles of 0 to $10 \mu$ size, no powder was obtained. Microscopic examination revealed the presence of a very small proportion of particles approximately $10 \mu$ in diameter and very few particles smaller than that. Therefore, it was impossible to collect a sample of fine particle size fulfilling the heretofore established requirement for solid phase spectroscopy, namely, that the particles be smaller than the wavelength of incident radiant energy in order to minimize scatter. 
The smallest particle size fraction of the bulk powder most easily obtainable for study was that passing the No. 325 sieve. This fraction will henceforth be designated as 325-mesh powder (less than 44- $\mu$ particles). Photomicrographs ${ }^{4}$ of this fraction, figure 1 , show that on the average the particles approximate spheres and their surfaces appear to have cusps and valleys.

Several unsuccessful attempts were made to produce a satisfactory powder of finer particle size. The mineral oil mull technique was employed and the resulting infrared spectrum, which was published in 1952 [10], exhibited evidences of degradation, showing absorption bands at 3 and $6 \mu$ and a broad, intense band from 8.4 to $12 \mu$. This degradation was the result of the prolonged mulling required to produce the requisite particle size, due to the hardness of the polymer. As mulling proved to be an unsatisfactory method of reducing particle size, grinding the sample in a cooled, vibratory ball mill [11] was attempted. The powder obtained, however, was discolored and its infrared spectrum. contained extraneous absorption bands. Figure 2 is a photomicrograph of the powder that was subjected to 3 hours' ball milling. The appearance of the particles is changed considerably by this treatment.

Another polyvinyl chloride sample, polymer $121,{ }^{5}$ was measured in the infrared to determine the influence of particle size on the spectrum. Polymer 121,

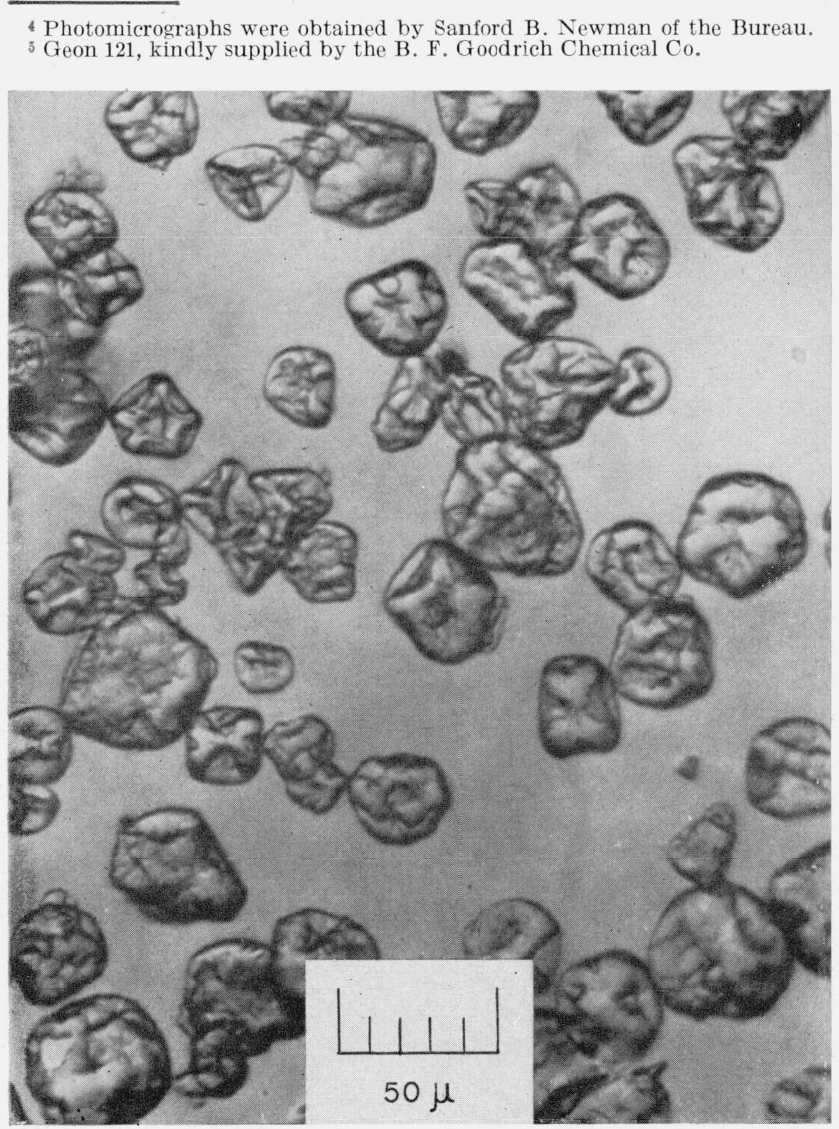

Figure 1. Photomicrograph of 325-mesh polyvinyl chloride.
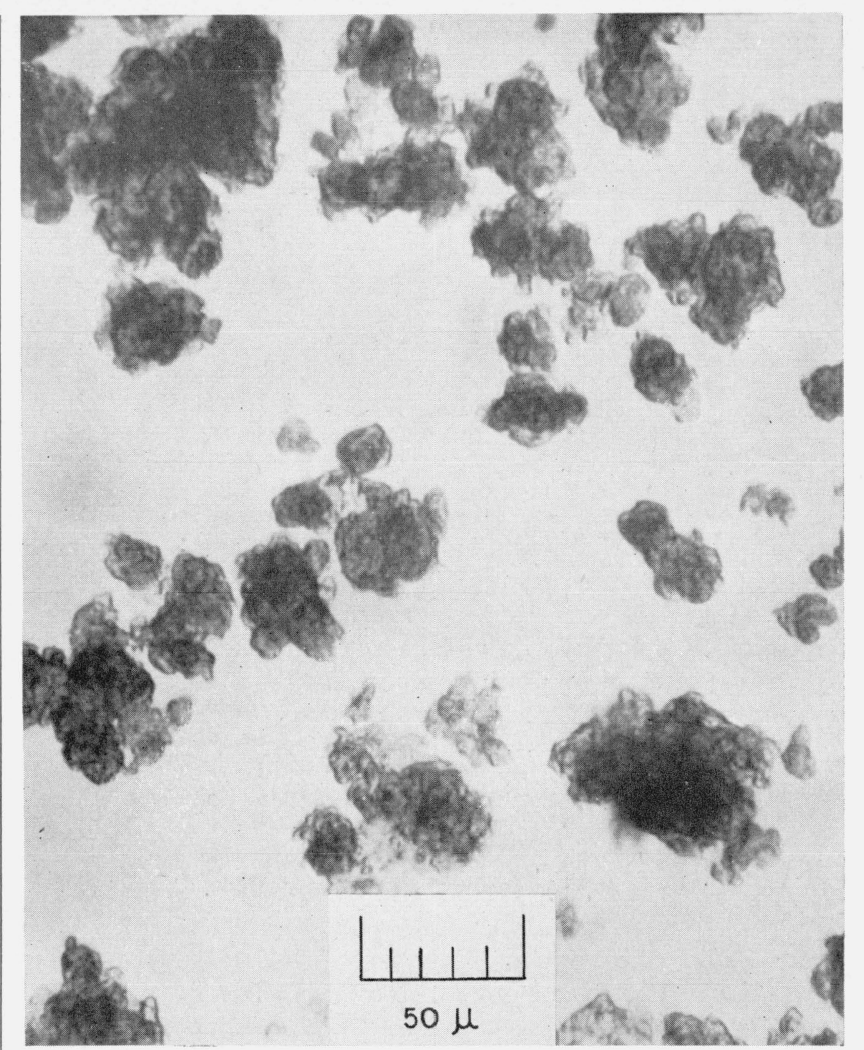

Figure 2. Photomicrograph of ball-milled polyvinyl chloride.

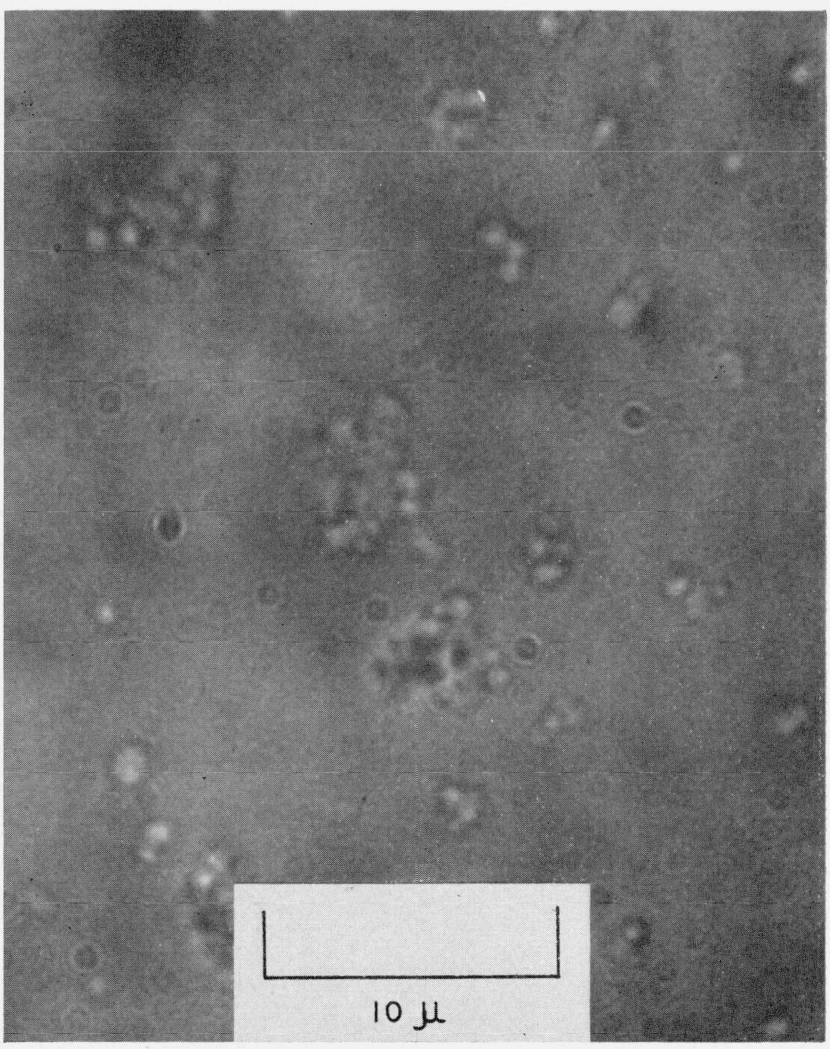

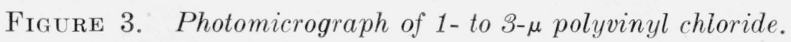


a paste resin, is a fine white powder of approximately 1- to $3-\mu$ particle size. Figure 3 is a photomicrograph of this powder.

\section{Suspension of Solids in Liquids}

Because of the deleterious effects of grinding the polymer, a method to obtain an infrared spectrum of polyvinyl chloride in the undegraded state was sought.

The 325-mesh polymer was mixed (not mulled) in mineral oil. The infrared spectrum of the resulting paste was quite good except in the range from 2 to $6 \mu$ where the radiation was badly scattered.

It was found that if, instead of mineral oil, a liquid was used for the mixing whose index of refraction more nearly matched that of the polymer, the scattering of the radiation could be eliminated.

The index of refraction of polymer 101 was measured in white light by grain immersion methods, using oils standardized in sodium light, and was found to be 1.548 at $27^{\circ} \mathrm{C}$. The oils used in the measurement are prepared by mixing various proportions of mineral oil whose index is $1.46 n_{D}^{20}$ and $\alpha$-monobromonaphthalene whose index is $1.66 n_{D}^{20}$. When the powder is observed in the liquid mixture of matching index of refraction, it is almost invisible. When the index of refraction of the liquid mixture differs appreciably from that of the powder, the powder is clearly discernible. An example of this latter situation is shown in figure 1 where the powder is seen against a background of mineral oil.

When the 1.54 and $1.56 n_{D}^{20}$ liquid mixtures were used with the 325-mesh polymer to obtain pastes suitable for infrared measurement, the resulting spectra showed the complete elimination of the scattering of the wavelengths from 2 to $15 \mu$. A spectrum obtained on a mix of powder and the $1.56 n_{D}^{20}$ liquid was also published in 1952 [10].

Apparently, only a near-match of indices is necessary to eliminate scatter, but an appreciable difference will not be tolerated. The $1.61 n_{D}^{20}$ immersion liquid was too poor a match for the powder and again scattering occurred. When perfluorokerosene (1.32 $\left.n_{D}^{20}\right)$, which has no absorption bands from 2 to approximately $7.3 \mu$ with the exception of a weak band at $4.2 \mu$, was used as the immersion liquid, scatter was extreme and there was not even an indication that there were bands in polyvinyl chloride around 3.4 and $7 \mu$.

$\alpha$-Monobromonaphthalene, however, has considerably more absorption bands in the infrared than does mineral oil. When the 325-mesh powder is suspended in the $1.56 n_{D}^{20}$ liquid and placed in a rock salt cell of minimum thickness, the infrared spectrum contains $\alpha$-monobromonaphthalene bands of appreciable intensity. Consequently, compensation must be made for the liquid in order to obtain the spectrum of the polymer. It is possible to achieve good compensation except in the regions where the liquid absorbs intensely, such as the 3.5- and the 12.5 - to $13.5-\mu$ regions. Here, inversion of the absorption bands of the liquid or other odd tracings may result [12].
Hexachlorobutadiene, with an index of refraction of $1.5542 n_{D}^{20}$, proved to be an excellent suspending liquid for the 2- to $6-\mu$ region, where it exhibits no absorption. Beyond $6 \mu$, however, several intense absorption bands are present which mar its usefulness. Hence, no single liquid suitable as a medium for the whole region from 2 to $15 \mu$ was found.

\section{Suspension of Solids in Solids}

The requirements of a satisfactory suspending medium for relatively large particles are: (1) The substance should be of nearly matching index of refraction, and (2) the substance should exhibit negligible absorption bands in the infrared region. A substance that fulfills the second requirement is potassium bromide. Potassium bromide has an index of refraction of $1.56 n_{D}^{20}$ and thus fulfills the first requirement as far as polyvinyl chloride is concerned. Potassium bromide, therefore, should be well suited for use with polyvinyl chloride and this was found to be the case.

The potassium bromide pellet technique $[1,2]$ was employed with several modifications of the method being made. It was desired to incorporate the 325mesh polymer particles in the potassium bromide without subjecting them to grinding. An intimate admixture of the two powders is required as each particle of polymer powder must be surrounded by potassium bromide so that upon application of pressure the potassium bromide will flow into the "pockets" of the polymer particles. In this way, a pellet with a homogeneous index of refraction is produced. Sufficient admixture was obtained when the polymer and potassium bromide powders were thoroughly stirred together with a spatula.

To obtain a spectrum with absorption bands of appreciable intensity, it was found in the case of polyvinyl chloride that a considerable quantity of sample was required. Pellets were prepared successfully in the $7 / 8$-in. diameter dies using 2 percent polymer; i. e., $0.028 \mathrm{~g}$ of polyvinyl chloride and 1.4 $\mathrm{g}$ of potassium bromide. The resulting pellets were 0.05 in. thick.

\subsection{Method and Equipment}

To prepare a satisfactory pellet with this quantity of material, all conditions must be carefully controlled. Fisher's C. P. grade potassium bromide is ground in an agate mortar and sieved through a 150-mesh sieve. ${ }^{5}$ Portions of $1.4 \mathrm{~g}$ are weighed into small aluminum cups and dried at $105^{\circ} \mathrm{C}$ for $100 \mathrm{hr}$ in an air oven. Polymer portions of $0.028 \mathrm{~g}$ are weighed into smaller aluminum cups and desiccated. First, an approximately 50:50 potassium bromide-polymer mixture is obtained by transferring some of a potassium bromide portion to a cup containing polymer and thoroughly mixing the two powders with a spatula. Then this 50:50 mixture is diluted by the bulk of the potassium bromide to yield a 2:100 polymer-potassium bromide mix-

${ }^{6}$ Since this writing, the Harshaw Chemical Co. has marketed 325-mesh potassium bromide of optical quality which is currently being used for the preparation of pellets in this laboratory. 
ture. This mixing process is carried out in a dry box at less than 3 -percent relative humidity ${ }^{7}$ as is the transfer of the powder mixture to the mold unit, parts $\mathrm{a}$ and $\mathrm{b}$ of figure 4 . Before adding the top plunger, the mold unit is tapped to settle the powder evenly over the surface of the lower plunger. A small rubber band is placed around the top plunger to suspend it above the powder and also above the vacuum outlet of the chase, b. The mold unit is removed from the dry box, placed on a hardened steel disk, c, on the press platen, surrounded by a rubber wall, d, which also has a vacuum outlet, and topped by another hardened steel disk, e. A seal is formed between the disks and the rubber wall by the application of a 200-lb load and a vacuum; the assembly at this stage is shown in figure $5, \mathrm{~A}$. When a vacuum of approximately $40 \mu \mathrm{Hg}$ is obtained, the load is applied slowly until a maximum pressure of approximately 95,000 psi is attained. The mold assembly under load is depicted in figure 5, B. The final pressure is held for $7 \mathrm{~min}$ and then slowly released. After release of the vacuum, the assembly can be separated and the pellet ejected from the mold unit by pressing the plungers and pellet out of the chase. A rod of smaller diameter than that of the plungers is used as an ejector and a corkfilled tube of diameter larger than that of the plungers as the receiving receptacle. This method of releasing the pellet without subjecting it to sudden changes in load prevents the formation of cracks in the pellet or of cleavage planes at its perimeter. Ejection of the pellet from the mold unit is depicted in figure 6 . The pellet is immediately placed on removal in a desiccator, over silica gel, until the infrared spectrum is measured. Good pellets can be made without evacuating the powder but the results are not as reproducible. Pellets of potassium bromide alone $(1.4 \mathrm{~g})$ were made for use as reference pellets.

Extensive experimentation with an earlier mold and an available vacuum chamber [13] aided in the design of the present simple setup. The requirements are:

1. Two hard cylinders with all surfaces mirror polished and having plane-parallel ends;

2. A hard confining wall fitting these two rods snug!y, provided with a vacuum outlet and with inner contacting wall mirror polished;

3. Two hard plane-parallel surfaces to protect the press platens;

4. A means of enclosing the parts of 1 and 2 , utilizing the parts of 3 as the top and bottom, so that the whole unit is evacuable.

Hardened Stentor tool steel was used for the necessary parts satisfying the requirements of points 1,2 , and 3 . To fulfill the fourth requirement, a wall was molded of natural rubber. ${ }^{8}$ It afforded a simple means of providing for the considerable amount of vertical travel that is necessary when forming thick

${ }^{7}$ At present, the relative humidity is approximately 1 percent as a result of improvements made in the dry box.

${ }^{8}$ The rubber was compounded with 10 parts of carbon black per 100 parts of rubber and cured for 1 hour. The product gave a Shore hardness value of 40 The wall was made in the Rubber Section of the Bureau.

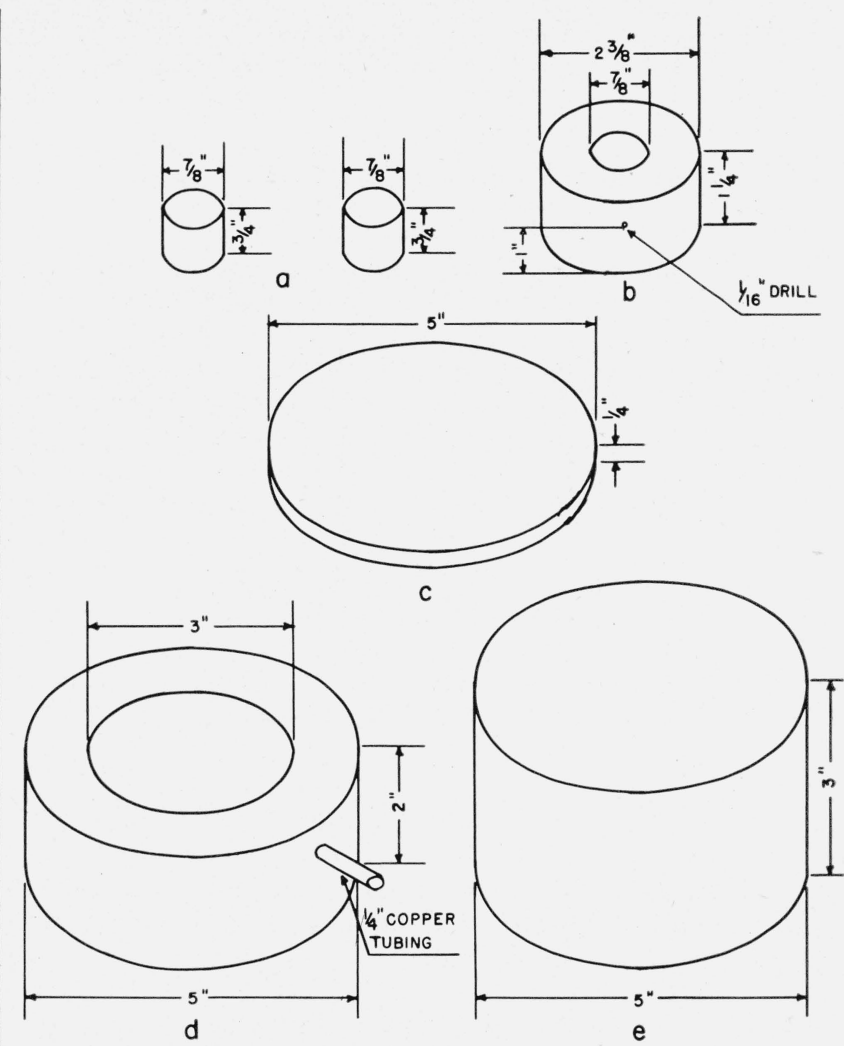

Figure 4. Components of the vacuum mold used for making $\mathrm{KBr}$ pellets.

a, Two steel plungers; b, enclosing steel chase; c, bottom steel disk; d, rubber wall; e, top steel disk.

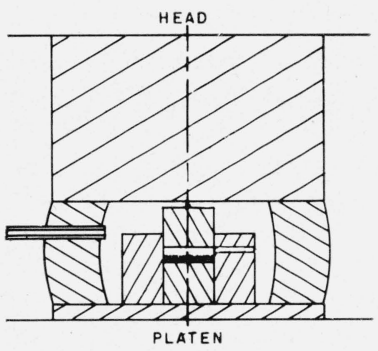

A

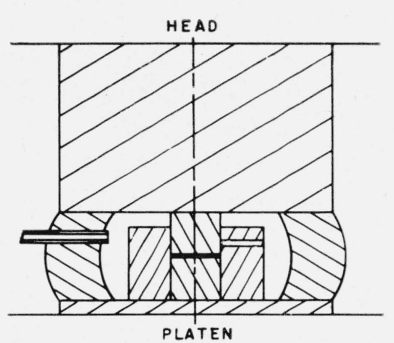

B
Figure 5. Mold assembly.

A, During evacuation; B, under load.

pellets. The wall thickness was 1 in. A hole was drilled through the wall and fitted with a brass tube as a vacuum outlet. The tube was sealed in the hole with a rubber adhesive. An improved design incorporates the vacuum outlet in the top steel disk and leaves the rubber wall as a solid piece.

A hydraulic testing machine capable of a maximum load of $60,000 \mathrm{lb}$ is used to press the pellets. A stationary head is empıoyed to insure application of the load perpendicular to the press platen because perfect alinement of all parts is essential at the high pressures used during pellet formation. 


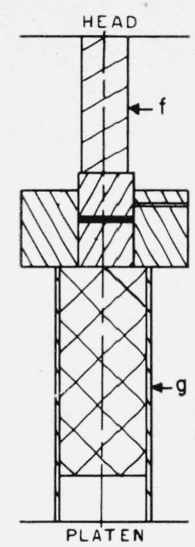

A

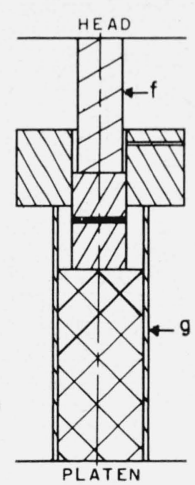

$B$

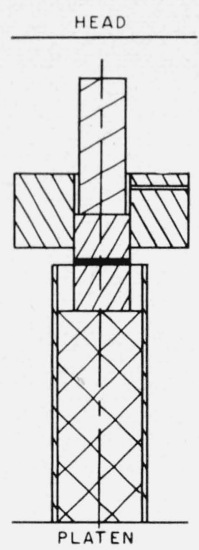

C

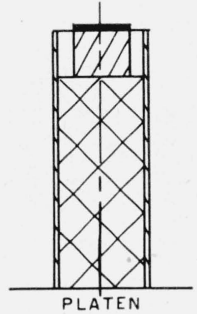

D
Figure 6. Removal of a pellet from the mold.

A, Setup for ejection, f, ejector rod, g, cork-filled ejector tube; B, pellet pressed free of chase; $\mathrm{C}$, load released; $\mathrm{D}$, pellet free.

A Perkin-Elmer model 21 double-beam infrared spectrophotometer with sodium chloride prism was used for measurements in the 2 - to $15-\mu$ region; in addition, some preliminary measurements were made with a cesium bromide prism in the PerkinElmer 21 in the spectral range from 15 to about $38 \mu$.

The pellets are larger than the infrared beam at the normal position of sample placement and therefore are merely placed in heavy paper holders fastened in place by two paper clips and the unit slipped into the cell mounts on the monochromator.

\section{Results and Discussion}

Potassium bromide pellets prepared with this equipment are transparent with the occasional exception of a cloudy edge, probably caused by a variation in leves of the powder layer in the mold unit. When the 325-mesh polymer particles are incorporated in the potassium bromide, the pellets are still transparent enough to read print through them when they are laid upon the printed matter. If the printed matter is placed about $3 \mathrm{ft}$ away, it can no longer be seen when viewed through the pellet (fig. 7). These pellets were made prior to the inclusion of the cork ejector in the pressing routine, hence a few cracks or strains may be noticed. Such slight imperfections can be tolerated becaue the pellets may be oriented in the holder in such a manner that the imperfections are not in the path of the infrared beam. This is possible because the pellets are $7 / 8$ in. in diameter and the beam is just less than $1 / 4$ by $3 / 4$ in. at the normal position of sample placement.

5.1. Spectrum of Relatively Large Particles of Polyvinyl Chloride in Potassium Bromide and in Liquids

The infrared spectrum of the sample pellet just described, compensated by the reference pellet, is shown as the solid curve in figure 8 . There is no

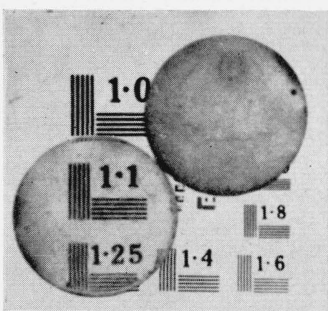

A

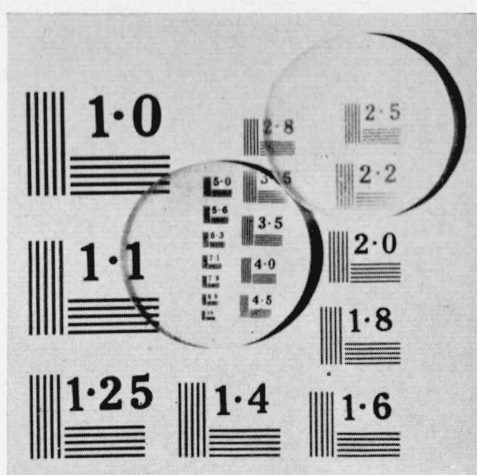

A

\section{B}

Figure 7. Pellets simulating distance viewing (left) and contact viewing (right).

$\mathrm{A}, \mathrm{KBr}$ reference pellet; $\mathrm{B}$, pellet of polyvinyl chloride in $\mathrm{KBr}$.

evidence of scattered radiation and the complete 2to $15-\mu$ region is measurable. In fact, potassium bromide is usable in the infrared as far as approximately $38 \mu$. Figure 9 shows the spectrum of the same sample pellet and also of the potassium bromide reference pellet in the $15-$ to $38-\mu$ region.

The other curves shown in figure 8 were obtained on mixtures of the 325-mesh polymer particles and various liquids; compensation for the liquid was made in each case. The use of these liquids was discussed previously. The noncompensable portions of the curves were deleted and the $1.56 n_{D}^{20}$ and $1.61 n_{D}^{20}$ liquid curves are not included beyond $6 \mu$ in order to avoid confusion.

\subsection{Spectrum of Potassium Bromide}

The infrared spectrum of an 0.05-in. thick pellet of potassium bromide is shown as a dotted line in figure 10. There is evidence of a slight amount of impurity in the potassium bromide as indicated by the $3.42-$ and $7.22-\mu$ bands. The absorption bands at 2.9 and $6.1 \mu$ can probably be attributed to water. These bands could not be reduced by drying the potassium bromide for a longer time in an air oven. If the potassium bromide were not dried at all (a sample freshly removed from the reagent bottle, ground, and immediately pressed), the bands were not appreciably increased but the radiation was scattered from 2 to $6 \mu$ and the pellet was translucent rather than transparent. The sample to be incorporated in the potassium bromide should also be dry or general scatter will result.

It is believed that the 2.9- and $6.1-\mu$ bands cannot be attributed to potassium hydroxide because a pellet pressed from fine potassium bromide that was freeze-dried from deuterium oxide showed bands at 3.99 and $8.33 \mu$, which could be completely eliminated by subjecting the powder to vacuum drying at $125^{\circ}$ C. However, evacuation alone would not accomplish this, and as the use of high temperature when the sample is involved is to be avoided, this method did not constitute an improved drying method. 


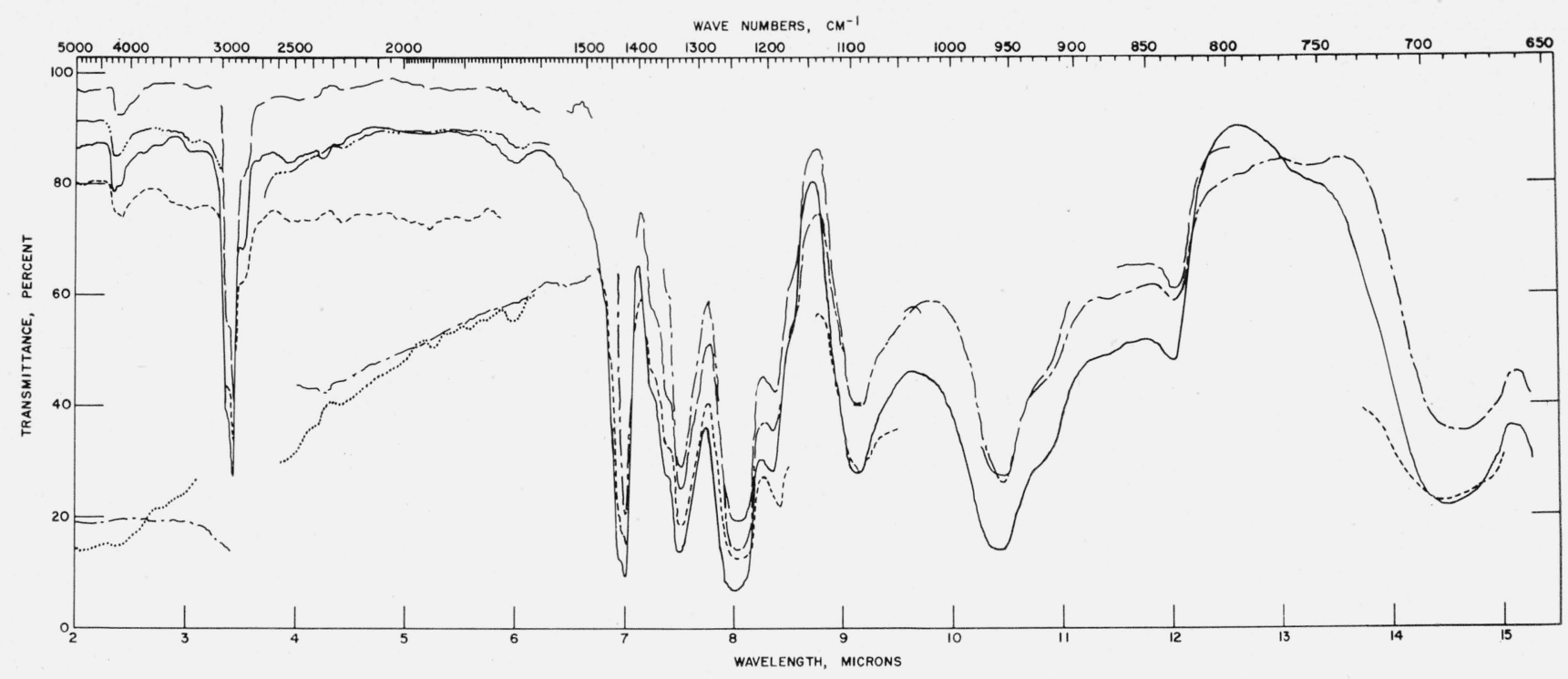

Figure 8. Infrared spectra of polyvinyl chloride powder.

Polymer particle size: $<44 \mu$. In each case the polymer was mixed with the suspending medium and compensation for that medium was made.

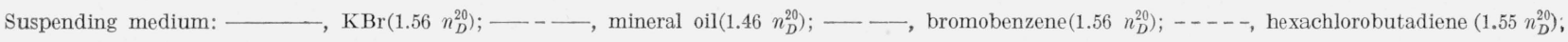
$\ldots-$, mineral oil and $\alpha$-monobromonaphthalene $\left(1.56 n_{D}^{20}\right) ; \cdots \cdots \cdots$, mineral oil and $\alpha$-monobromonaphthalene $\left(1.61 n_{D}^{20}\right)$.

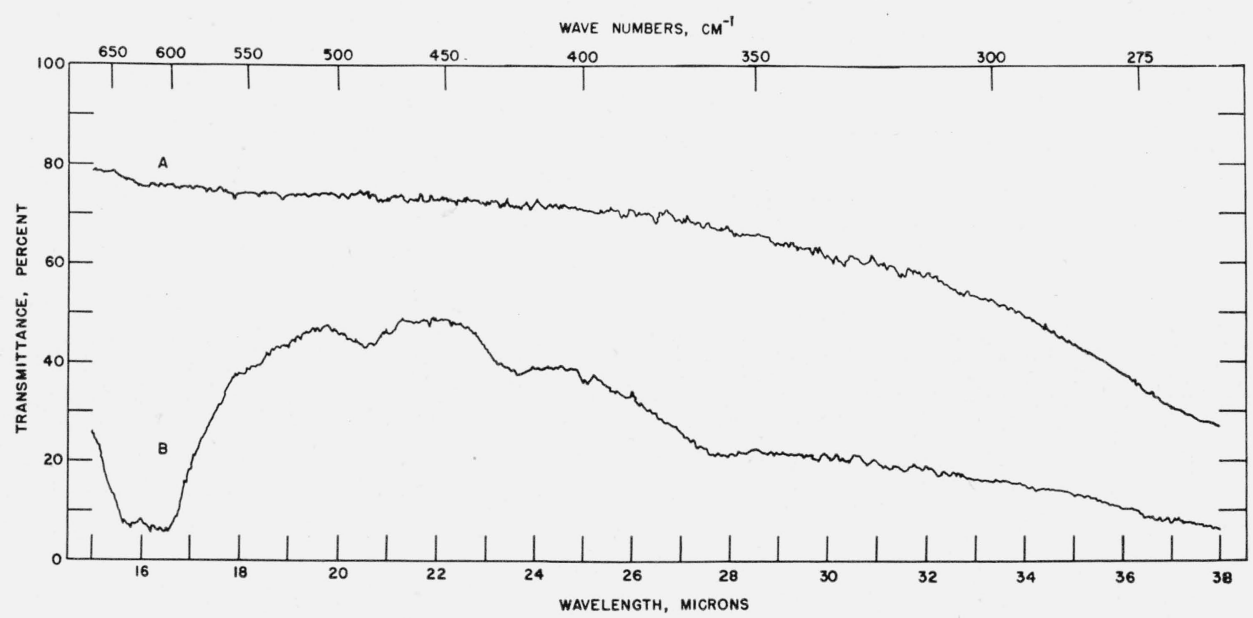

FIGURE 9. Long wavelength infrared spectra of polyvinyl chloride and $\mathrm{KBr}$ pellets.

A, $1.4 \mathrm{~g} \mathrm{KBr} ; \mathrm{B}, 1.4 \mathrm{~g} \mathrm{KBr}+0.028 \mathrm{~g}<44 \mu$ particles of PVC.

The broad absorption bands centering around 6.95 and $8.8 \mu$ are probably introduced during the grinding operation performed on the potassium bromide. In a series of experiments, a sample of potassium bromide was ground in a mullite mortar, pressed into a pellet, and the spectrum measured. The pellet was then reground in the mortar, repressed, and the spectrum measured. The whole process was repeated a third time. Each succeeding spectrum showed a band around $9.2 \mu$, of the same shape and of increased intensity after each grinding. This band, therefore, can be attributed to contamination of the potassium bromide resulting from abrasion of the mortar.

It was found that an agate mortar introduced less impurity than one of either mullite or alumina. It is possible that the agate mortar shows less tendency to abrade because its surface is highly polished. The spectrum shown in figure 10 is that of a pellet made from agate-ground potassium bromide. ${ }^{9}$

\footnotetext{
${ }^{9}$ The pellets that are currently being made in this laboratory with the 325 -mesh optical quality Harshaw potassium bromide and prepared in the approximately 1-percent relative humidity dry box, show a much cleaner spectrum with such small absorption bands due to water that it is now not necessary to compensate for the potassium bromide.
} 


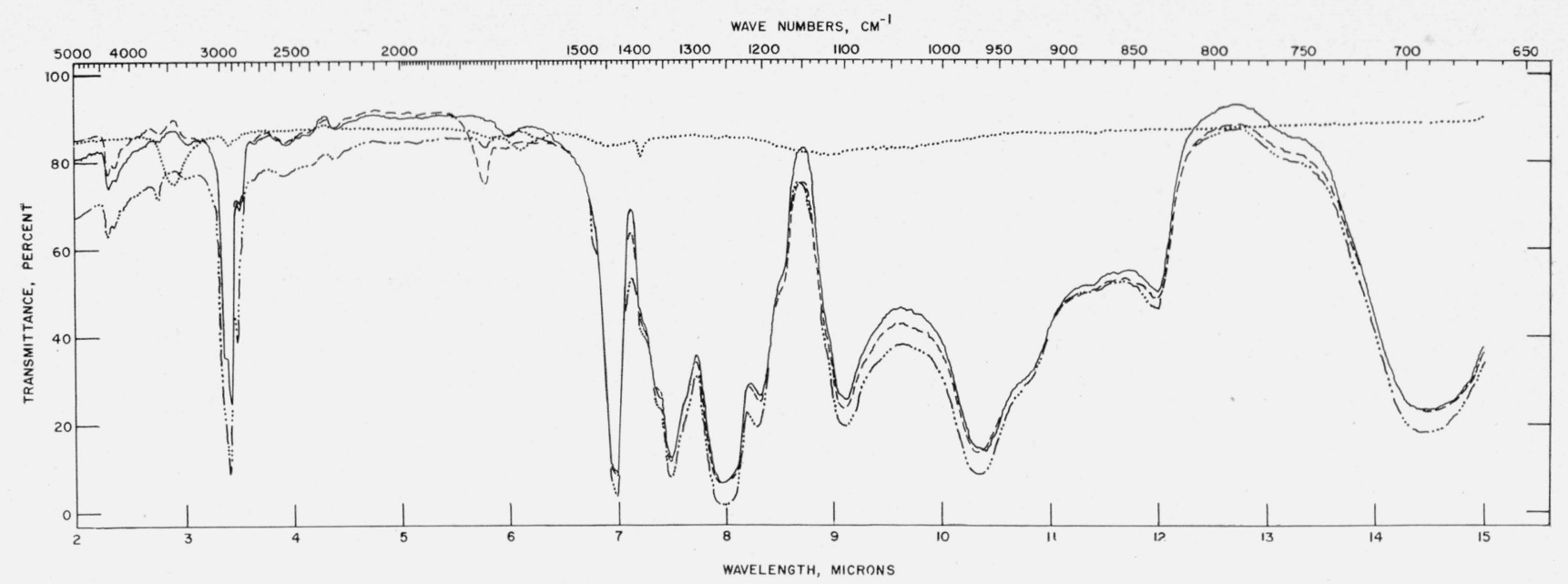

FIGURE 10. Infrared spectra of polyvinyl chloride-KBr pellets.

$1.4 \mathrm{~g} \mathrm{KBr}$ (1 to $3 \mu$ particles)

.028 g PVC compensated by 1.4 g K Br: untreated PVC ( $<44 \mu$ particles $)-$ ; ball-milled PVC-_-_-; untreated PVC

\subsection{Spectrum of Ball-Milled Polyvinyl Chloride in Potassium Bromide}

The solid curve in figure 10 presents the compensated spectrum of a pellet containing untreated polymer 101 of relatively large particle size (polymer passing the 325-mesh sieve). The compensation of the potassium bromide is particularly noticeable in the water regions of 2.9 and $6.1 \mu$. This spectrum is presented here for comparison with that of polymer 101 subjected to 3 hours' ball milling prior to its incorporation in a pellet. The latter spectrum is shown in figure 10 as a dashed line which closely follows the solid line with the notable exception of the absorption band introduced at $5.78 \mu$. As was previously stated, the ball milling caused discoloration and changes in particle configuration. The presence of the $5.78-\mu$ band constitutes further proof of the inadvisability of attempting comminution of the polymer in any way prior to measurement when a spectrum of the polymer in an undegraded state is desired.

\subsection{Spectrum of Fine Particles of Polyvinyl Chloride in Potassium Bromide}

Also shown in figure 10 is the spectrum of polymer 121, the paste resin composed of particles 1 to $3 \mu$ in size (refer to fig. 3). The curve is similar to that representing the 325-mesh particles of polymer 101 . Some differences in the two curves are probably due to the methods of preparing each polymer, e. g., the small bands present at 2.75 and $5.8 \mu$ in the spectrum of Geon 121. The success in obtaining a spectrum of the relatively large particles so similar to the spectrum of very small particles is attributed to the apparent match in indices of refraction of the polymer and potassium bromide.

\subsection{Index of Refraction Match of Polyvinyl Chloride and Potassium Bromide}

The apparent match of indices of refraction of the polymer and potassium bromide was investigated. The indices of refraction of potassium bromide have been measured in the ultraviolet, visible, and/or infrared regions of the spectrum by several authors: Gyulai [14], Gundelach [15], Forrest [16], and Stephens, Plyler, Rodney, and Spindler [17]. However, the only reported measurements of the index of refraction of polyvinyl chloride were made in the visible. Schildknecht [18] reports a value of 1.54 for commercial unplasticized vinyl chloride resins and Fuoss [19] reports a value of 1.565 obtained at $40^{\circ} \mathrm{C}$. The refractive index of polymer 101 was measured and found to be 1.548 at $27^{\circ} \mathrm{C}$. This value nearly matches the $1.56 n_{\mathrm{D}}$ of potassium bromide.

Methods for determining the infrared refractive indices of polyvinyl chloride were sought without much success. Determination by the prism method was not tried because it was felt that a prism of polyvinyl chloride will not be sufficiently transparent to infrared radiant energy. Efforts were made to calculate the index of refraction at $2 \mu$ from the infrared spectrum of a thin film of the polymer. A film that exhibited interference fringes in the infrared spectrum between 2 and approximately $5 \mu$ was selected. The index of refraction was calculated using the following equation:

$$
n \lambda=2 t \eta \cos \theta,
$$

where $n=$ fringe number, $\lambda=$ wavelength, $t=$ thickness, $\eta=$ index of refraction, and $\cos \theta=$ cosine of the angle of incidence, taken to be 1 . The thickness of the film, $t$, was determined by measuring the 
distance between two flat calcium fluoride windows separated by a spacer of the film, following the method described by Smith and Miller [20]. A refractive index of 1.51 at $2 \mu$ was calculated for the polymer by this method, which is admittedly subject to several errors.

The elimination of scattering of the infrared radiant energy when the polymer is pelleted with potassium bromide indicates that the infrared refractive indices of polyvinyl chloride, like the visible indices, approximate those of potassium bromide. In fact, the elimination of scatter appears to be a method for estimating the infrared indices of refraction of the polymer. Further study was undertaken to substantiate this method.

It is known that the presence of ultraviolet and visible electronic absorption bands in a material results in a characteristic variation of refractive index for wavelengths in the neighborhood and interior of the band. The shape of the resultant dispersion curve is described by Lorentz's equation. The large change in refractive index produces the phenomenon of Christiansen windows $[21,22]$. However, in the infrared spectral region absorption bands are associated with molecular vibrations. Not many dispersion curves have been determined for covalent compounds but from those available it is clear that, although some bands are associated with large refractive index changes, many bands affect dispersion slightly or not at all $[23,24]$. Price and Tetlow [22] have suggested the same conclusion from their observation of Christiansen windows in infrared spectra of mulled organic samples. Moreover, the aopearance of the Christiansen filter effect in mulled organic samples has been noted in relatively few cases, and in fact, there was no indication of the presence of this effect when polyvinyl chloride was mixed with any of the various liquids mentioned in part 3 . Hence, it might be hoped that the index of refraction of polyvinyl chloride will slowly decrease as longer wavelengths are ap- proached, but that few large variations in the vicinity of absorption bands will be present.

Figure 11 presents the refractive indices of various salt crystals for the visible and infrared region of the spectrum. ${ }^{10}$ There is some change in the indices of these salts from the visible to $2 \mu$ but little change from $2 \mu$ through the infrared region of interest.

Figure 12 presents the infrared spectra obtained by measuring pellets of three of these salts, each containing $0.028 \mathrm{~g}$ of 325 -mesh polymer 101 ; compensation was made for the salt in each case. When potassium bromide was used as the suspending medium, the spectrum was far superior. All sample pellets were of good quality and with compensation

\footnotetext{
10 The plot is adapted from the data presented by Joos [25].
}

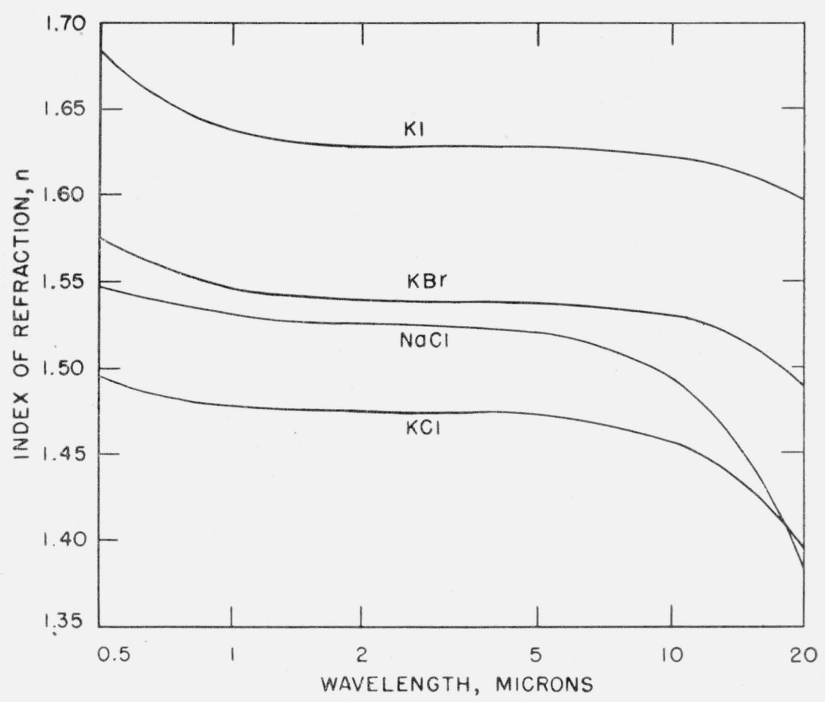

Figure 11. Refractive indices of synthetic crystals.

Adapted from data of G. Joos [25].

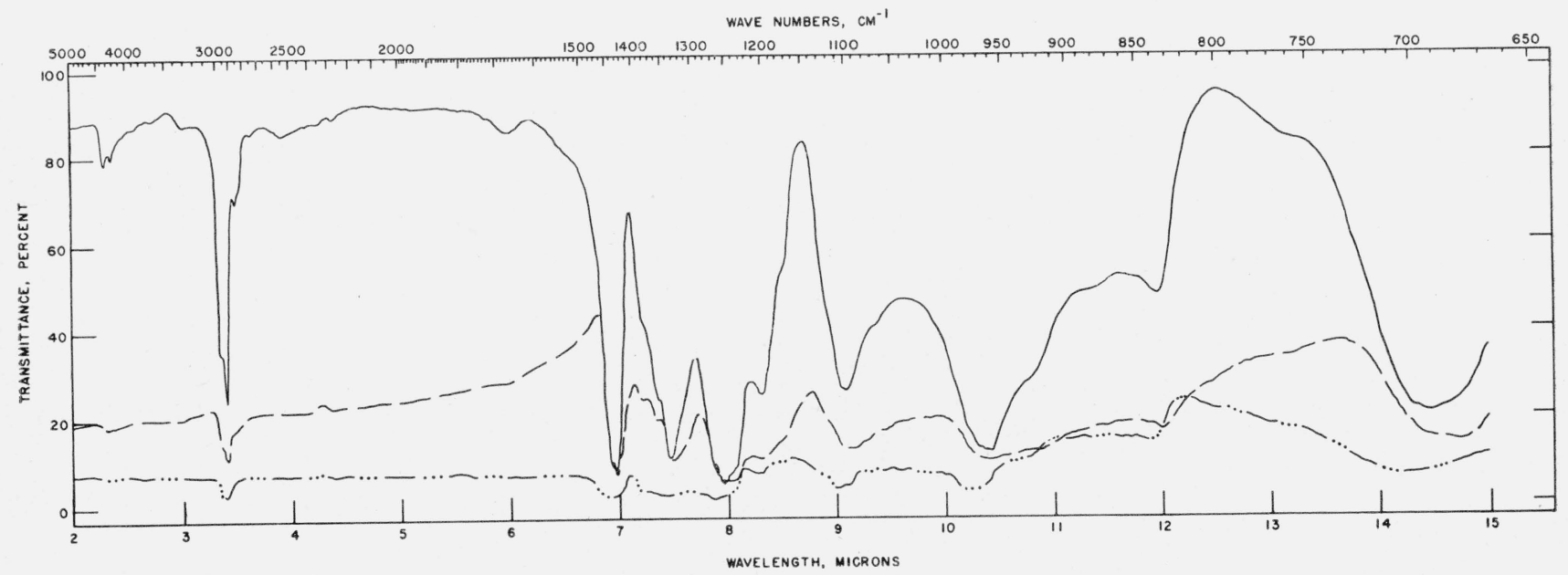

FIGURE 12. Infrared spectra of polyvinyl chloride-salt pellets. 
employed to eliminate impurities of the individual salts, spectra of equal quality should have been obtained if it were not a necessity to achieve a match of indices of refraction between relatively large sample particles and suspending medium. It is to be noted that although general scatter is extreme for the potassium chloride pellet. only the polymer band at $7 \mu$ shows a Christiansen effect. 'This is evidence that most poly vinyl chloride absorption bands do not have rapidly changing refractive indices. The potassium bromide pellet does not show a Christiansen window even at $7 \mu$.

\subsection{Necessity of Matching Indices of Refraction of Medium and Sample When the Sample Particles Are Relatively Large}

A series of pellets was prepared using potassium chloride as the imbedding salt or matrix and incorporating other salts (potassium bromide, potassium iodide, and sodium chloride) with a range of refractive indices in order to demonstrate the necessity for matching indices of refraction of a sample with its imbedding medium. The samples of the salts to be incorporated in the potassium chloride were selected so that the particles were relatively large (325 mesh). None of these salts absorbs in the 2 - to $15-\mu$ infrared region to an appreciable extent.

Thus, each pellet of the series is composed of two transparent materials and should yield a transparent pellet unless the mismatch in their indices of refraction causes scattering. Scattering does result in each case and increases with mismatch of the index of refraction of the sample salt and that of the potassium chloride. The uncompensated spectra of these mixed-salt pellets are shown in figure 13. The spectrum of sodium chloride in potassium chloride shows less scatter at the longer wavelengths where the index of refraction curves for these two salts are approaching each other (see fig. 11). It is, therefore, apparent that relatively large, solid particles can only be measured in the infrared when they are imbedded in a substance of nearly matching indices of refraction; otherwise, scattering of the radiant energy will result and obscure the spectrum.

\subsection{Effect of Sample Particle Size When the Indices of Refraction of Sample and Medium Are Matched}

To determine the effect of particle size on the infrared spectrum when the indices of refraction of sample and matrix are nearly matched, another series of pellets was prepared. Polyvinyl chloride was used as the sample and potassium bromide was selected as the imbedding salt as it was shown to afford the best match in indices of refraction with the polymer (see fig. 12). Figure 14 presents the compensated spectra of five pellets of polyvinyl chloride. The same weight of polymer, $0.028 \mathrm{~g}$, was pelleted in each case. The dotted curve was obtained with polymer 121 and all others with polymer 101. It is of interest to observe that only the smallest particles display evidence of scatter at short wavelengths. It is known that scatter is maximum for particle diameters about equal to twice the wavelength, but for larger particles scatter varies from about half to about three-fourths of the maximum value [26]. The only significant change in the spectrum as the particle size is decreased appears to be the increase in intensity of the absorption bands.

Figure 15 shows a plot of the strong polyvinyl chloride band at $8 \mu$ as a function of predominant particle size. The experimental points were obtained from the data shown in figure 14, and the solid line is a theoretical curve [12] for this band based on an absorption coefficient of $1,000 \mathrm{~cm}^{-1}$ as estimated from a spectrum of polymer 101 film. Calculations were made under the assumption of zero scatter and uniform particle size. The agreement is satisfactory.

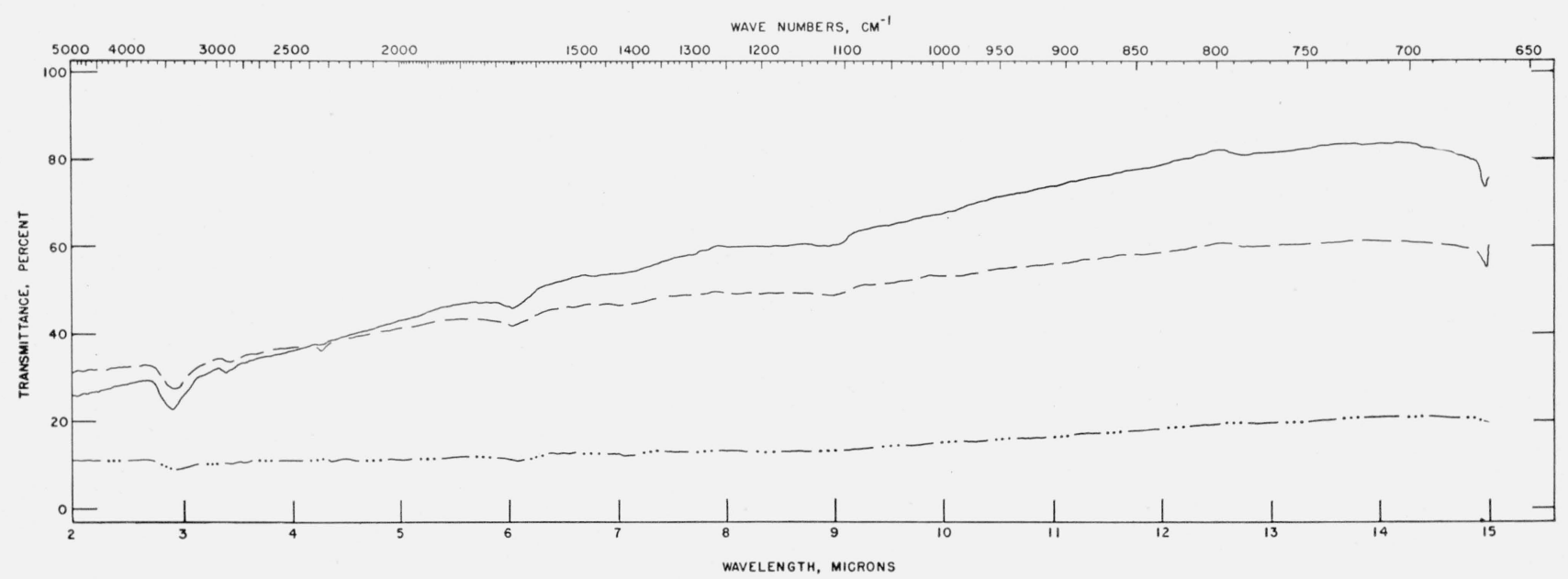

Figure 13. Infrared spectra of salt- $\mathrm{KCl}$ pellets.

Sample salt particle size $<44 \mu .1 .4 \mathrm{~g} \mathrm{KCl}+0.028 \mathrm{~g}$ sample salt: ——— $\mathrm{NaCl}$ in $\mathrm{KCl} ;----, \mathrm{KBr}$ in $\mathrm{KCl} ;--\cdots-, \mathrm{Kl}$ in $\mathrm{KCl}$. 


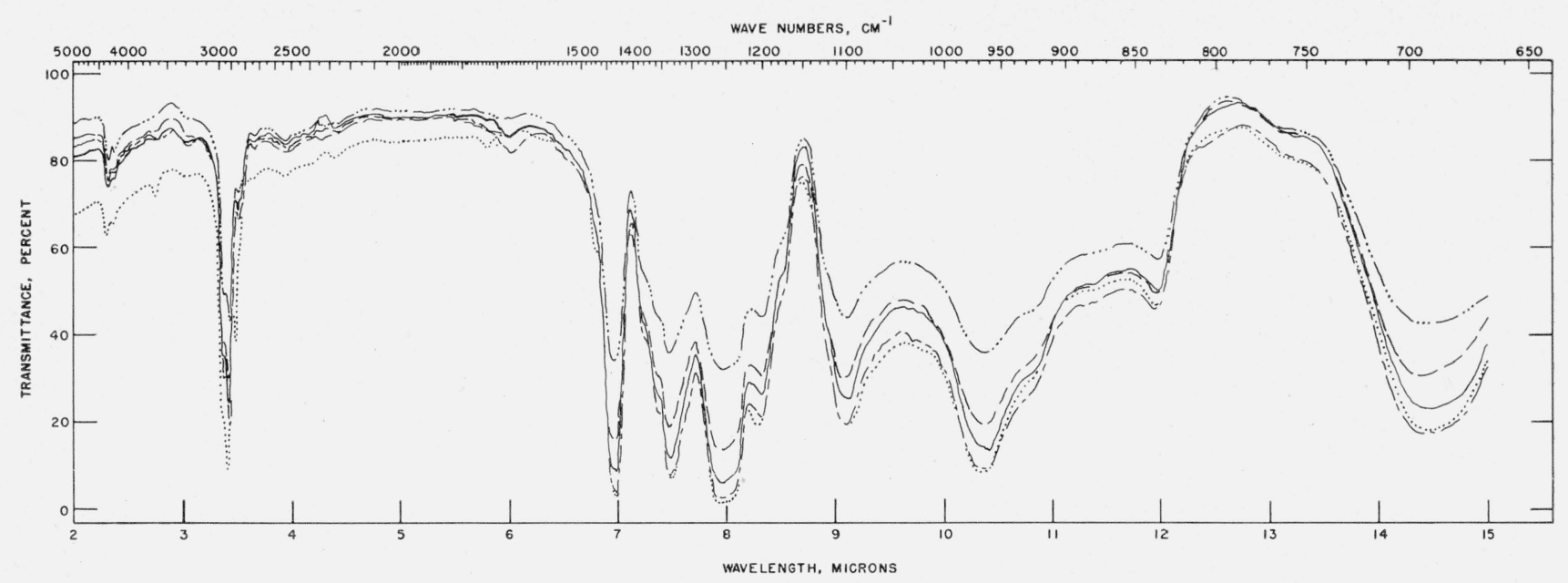

FiguRE 14. Infrared spectra of polyvinyl chloride-KBr pellets.

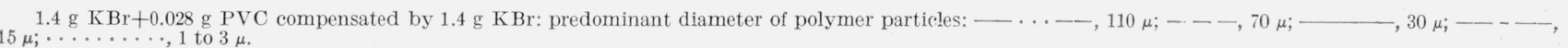

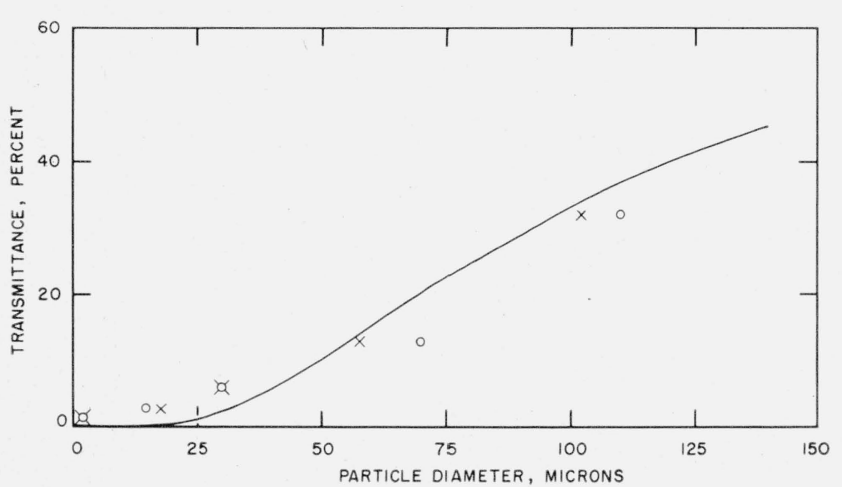

FIGURE 15. Influence of particle size on the polyvinyl chloride band ai 8 microns.

$1.4 \mathrm{~g} \mathrm{KBr}+0.028 \mathrm{~g}$ PVC pellet. $\bigcirc$, Predominant particle size; $\times$, median of particle size; - - , theoretical relationship between particie size and transmissior'.

\subsection{Spectral Measurements of Paper and Nylon Fibers}

Because very large particles can be measured to obtain a credible spectrum, it should be possible to measure fibers of appreciable diameter or other solid substances that cannot be reduced to a fine particle size. For example, pellets of nylon fibers and of paper fibers were prepared, both using potassium bromide as the suspending medium; the choice of matrix was indicated by their indices of refraction in the visible. Figure 16, curve A, shows the compensated spectrum of pelleted paper fibers, which had been ground in a Kerner mill. The fibers are approximately 5 to $30 \mu$ in diameter with the average diameter being about $10 \mu$; the length of the fibers is of the order of $400 \mu$. Curve B of this figure shows the compensated spectrum of pelleted nylon fibers, approximately $30 \mu$ in diameter and microtomed in approximately $30-\mu$ lengths.

The spectra of these two pelleted fibers are characteristic of spectra of their polymers and are identifiable as such.

\section{Conclusion}

The scope of solid phase infrared spectrocopy is broadened by the elimination of particle size restrictions heretofore placed on the sample. This applies only, however, to samples whose indices of refraction change only slightly with change in wavelength and can be matched to the indices of refraction of the suspending medium. The salts mentioned cover quite a range of refractive indices; when other crystals and numerous liquids that have infrared "windows" are taken into account, this range may be appreciably extended.

The authors express their appreciation to Francis A. Smith, Charles F. Bersch, and Walter K. Stone for valuable discussions and assistance during the conduct of the work, and to Harriet A. Baker and Margaret F. Toompas for preparation of the figures.

\section{References}

[1] M. M. Stimson and M. J. O'Donnell, The infrared and ultraviolet absorption spectra of cytosine and isocytosine in the solid state, J. Am. Chem. Soc. 74 , 1805 (1952).

[2] U. Schiedt and H. Reinwein, On the infrared spectroscopy of amino acids. I. A new preparation technique for infrared spectroscopy of amino acids and other polar compounds, Z. Naturforsch. [B] 7,270 (1952); U. Schiedt, II. An improvement on a preparation technique for the infrared photometric determination of amino acids and other polar compounds, ibid. [B] 8, 66 (1953). See also U. Schiedt (reported by H. Hausdorff), German technique for presset potassium bromide infrared sampling, Appl. Spectroscopy \%, 75 (1953).

[3] J. M. Hunt, M. P. Wisherd, and L. C. Bonham, Infrared absorption spectra of minerals and other inorganic compounds, Anal. Chem. 221478 (1950).

[4] M. Dolinsky, A technique for infrared analysis of solids insoluble in nonpolar solvents, J. Assoc. Offic. Agr. Chemists 34, 748 (1951). 


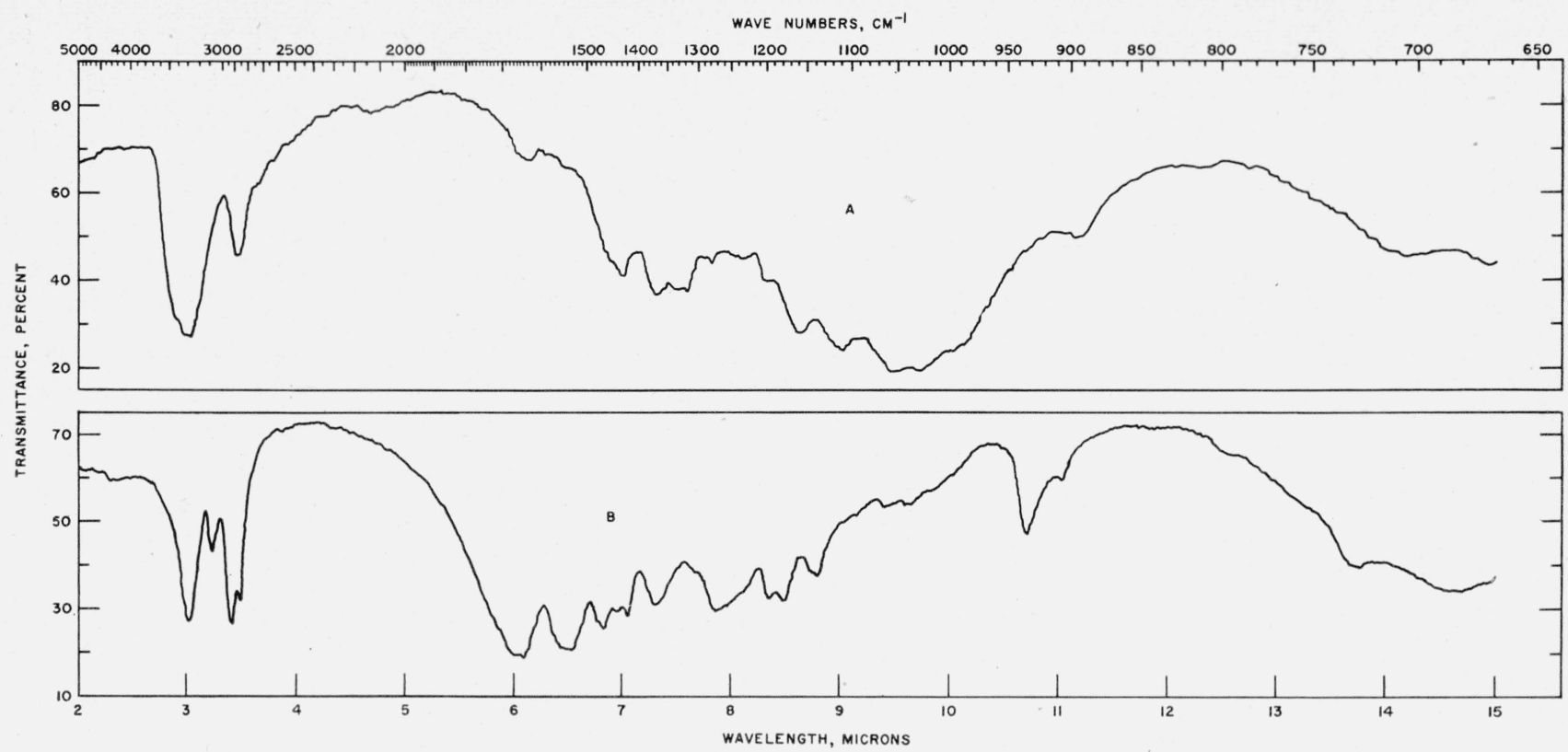

Figure 16. Infrared spectra of fibers pelleted in $\mathrm{KBr}$.

$\mathrm{A}, 1.4 \mathrm{~g} \mathrm{~K} \mathrm{Br}+0.0050 \mathrm{~g}$ paper compensated by $1.4 \mathrm{~g} \mathrm{KBr} ; \mathrm{B}, 1.4 \mathrm{~g} \mathrm{KBr}+0.0085 \mathrm{~g}$ nylon compensated by $1.4 \mathrm{~g} \mathrm{KBr}$.

[5] J. E. Tyler and S. A. Ehrhardt, Infrared spectra of evaporated films, Anal. Chem. 25, 390 (1953).

[6] J. D. Sands and G. S. Turner, New developments in solid phase spectroscopy of elastomers and plastomers, Anal. Chem. 24, 791 (1952).

[7] J. Lecomte, Studies in infrared spectra, Proc. Intern. Congr. Pure and Appl. Chem. 11th, London, I, 509 (1947)

[8] D. L. C. Jackson, Degradation of vinylidene chloride copolymers in solution, J. Soc. Chem. Ind., Trans. (London) 68, 247 (1949).

[9] P. S. Roller, Separation and size distribution of microscopic particles - an air analyzer for fine powders, U. S. Bureau of Mines Tech. Paper No. 490 (1931).

[10] B. G. Achhammer, Polymer degradation studies by absorption and mass spectrometry, Anal. Chem. 24, 1925 (1952).

[11] F. H. Forziati, W. K. Stone, J. W. Rowen, and W. D. Appel, Cotton powder for infrared transmission measurements, J. Research NBS 45, 109 (1950) RP2116.

[12] J. E. Stewart, Absorption of radiant energy by solid particles in suspension, J. Research NBS 54, 41 (1955) R P2563.

[13] L. A. Wood, N. Bekkedahl, and F. L. Roth, Measurement of densities of synthetic rubbers, J. Research NBS 29, 391 (1942) RP1507; Ind. Eng. Chem. 34, 1291 (1942).

[14] Z. Gyulai, The dispersion in the ultra-violet of a few alkali halides, Z. Physik 46, 80 (1927).

[15] E. Gundelach, The dispersion of potassium bromide crystals in the infra-red Z. Physik 66, 775 (1930).

[16] J. W. Forrest, Refractive index values for potassium bromide, J. Opt. Soc. Amer. 32, 382 (1942).
[17] R. E. Stephens, E. K. Plyler, W. S. Rodney, and R. J Spirdler, Refractive index of potassium bromide for infrared radiant energy, J. Opt. Soc. Amer. 43, 110 (1953)

[18] C. E. Schildknecht, Vinyl and Related Polymers, p. 429 (John Wiley \& Sons, Inc., New York, N. Y., 1952).

[19] R. M. Fuoss, Electrical properties of solids. 'V. Their variation at $40^{\circ} \mathrm{C}$ with frequency and composition in the system polyvinyl chloride-tricresyl phosphate, J. Am. Chem. Soc. 61, 2334 (1939).

[20] D. C. Smith and E. C. Miller, Infrared absorption cells and measurement of cell thickness, J. Opt. Soc. Amer. 34, 130 (1944).

[21] R. B. Barnes and L. G. Bonner, The Christiansen filter effect in the infrared, Phys. Rev. 49, 732 (1936).

[22] W. C. Price and K. S. Tetlow, Infra-red Christiansen filter effect in slurries of organic crystals, J. Chem. Phys. 16, 1157 (1948).

[23] A. H. Pfund, The dispersion of $\mathrm{CS}_{2}$ and $\mathrm{CCl}_{4}$ in the infrared, J. Opt. Soc. Amer. 25,, 351 (1935).

[24] M. A. Pittman Infrared dispersion of $\mathrm{CHCl}_{3}$ and $\mathrm{CHBr}_{3}$, J. Opt. Soc. Amer. 29, 358 (1939).

[25] G. Joos, Physics of Solids, Part II, p. 164 (Office of Military Government for Germany, Wiesbaden, Germany, 1948).

[26] B. Goldberg, New computation of the Mie scattering functions for spherical particles, J. Opt. Soc. Amer. 43, 1221 (1953).

Washington, December 28, 1955. 